\title{
Nuclear Spin Relaxation Parameters of MRI Contrast Agents - Insight from Quantum Mechanical Calculations
}

\author{
Oleg V. Yazyev ${ }^{[a]}$ and Lothar Helm ${ }^{*[b]}$
}

Keywords: Relaxivity / Hyperfine interactions / Quantum chemistry / Gadolinium / MRI contrast agent

Nuclear magnetic relaxation in the presence of paramagnetic centres has gained increasing interest in recent years partly due to its importance for contrast agents in magnetic resonance imaging. Rational design of new more efficient agents is possible as a result of a better understanding of the underlying relaxation mechanisms. Quantum chemical calculations together with molecular dynamics simulations allow obtaining fundamental parameters such as quadrupole coupling constants and hyperfine interaction tensors directly at a molecular level. Recent results are presented on gadolinium(III) ions in aqueous solution and on $\left[\mathrm{Gd}(\mathrm{DOTA})\left(\mathrm{H}_{2} \mathrm{O}\right)\right]^{-}$, a commercial MRI contrast agent. Isotropic hyperfine coupling constants can be calculated for ${ }^{17} \mathrm{O}$ and ${ }^{1} \mathrm{H}$ nuclear spins of water molecules in the first and second coordination sphere of $\mathrm{Gd}^{3+}$. It is also shown that the commonly used point-dipole approximation for the dipolar interaction between the electron and the nuclear spin is in general valid for ${ }^{1} \mathrm{H}$ spin but not for the directly bound ${ }^{17} \mathrm{O}$ spin. The calculated quadrupole coupling parameters allow a direct determination of the rotational correlation time of complexes from the ${ }^{17} \mathrm{O}$ nuclear spin relaxation.

(@ Wiley-VCH Verlag GmbH \& Co. KGaA, 69451 Weinheim, Germany, 2008)

\section{Introduction}

Studying relaxation phenomena in nuclear magnetic resonance (NMR) has become a major tool in physics and physical chemistry to study structure and dynamics in solution and in the solid state. This is because the macroscopically measured quantities, mainly the relaxation times $T_{1}$ and $T_{2}$, depend, at least in the most common cases, on the strength and temporal as well as spatial fluctuation of the local magnetic field at the nucleus observed. Bloembergen, Purcell and Pound showed already in 1948 that the relaxation of ${ }^{1} \mathrm{H}$ nuclear spins in water is associated to the local Brownian motion. ${ }^{[1]}$ What Bloembergen et al. described is essentially relaxation through dipolar coupling. Relaxation by other mechanisms like scalar coupling and quadrupolar coupling have been discovered later. ${ }^{[2]}$ All of them link local strength and fluctuation of properties such as magnetic field or electric field gradients to measurable relaxation rates. The study of nuclear spin relaxation rates therefore provides information on local structure and dynamics in solution.

[a] Institut de Théorie des Phénomènes Physiques, Ecole Polytechnique Fédérale de Lausanne, and Institut Romand de Recherche Numérique en Physique des Matériaux (IRRMA), EPFL$\mathrm{PPH}$,

1015 Lausanne, Switzerland

Fax: +41-21-693-9875

E-mail: oleg.yazyev@epfl.ch

[b] Institut des Sciences et Ingénierie Chimiques, Ecole Polytechnique Fédérale de Lausanne, EPFL-BCH,

1015 Lausanne, Switzerland

Fax: +41-21-693-9875

E-mail: lothar.helm@epfl.ch
After the discovery of magnetic resonance imaging (MRI), NMR expanded its area of application from basic science disciplines like physics and chemistry to medical diagnostics. MRI images are recorded from signals of ${ }^{1} \mathrm{H}$ nuclear spins of soft tissues. The contrast of the images depends on several parameters, among which longitudinal $\left(T_{1}\right)$ and transverse $\left(T_{2}\right)$ relaxation times are very important. ${ }^{[3]}$ Both, $T_{1}$-weighted and $T_{2}$-weighted images can be measured and give complementary information. An important step to augment information from MRI images was the advent of paramagnetic contrast agents in the mid1980s. ${ }^{[4,5]}$ As a result of interactions between the nuclear spins of water protons and unpaired electrons of a paramagnetic centre, these pharmaceutical compounds increase $T_{2}$ and/or $T_{1}$ relaxation times by different amounts.

In the last twenty years, development of new, more efficient contrast agents became an important aim in biological inorganic chemistry. ${ }^{[6]}$ Because of the inherent low sensitivity of MRI imaging, compared, for example, with optical and nuclear imaging methods, a primary goal of the research is to find new contrast agents with high efficiency. Efficiency is normally expressed in terms of relaxivity, which is the increase in the relaxation rate per millimolar concentration of the contrast agent added. ${ }^{[7,8]}$ To achieve a rational design of new compounds with higher relaxivity, a basic understanding of the underlying physicochemical parameters is inevitable. A main experimental tool is the measurement of relaxivity over a wide range of static magnetic fields at variable temperature. The results obtained are commonly named as nuclear magnetic relaxation dispersion 
(NMRD) profiles and can be fitted by theoretical models. ${ }^{[9-11]}$ Other experimental techniques are oxygen-17 $\mathrm{NMR},{ }^{[7]}$ deuterium NMR, ${ }^{[12]}$ and electron spin resonance spectroscopy. ${ }^{[13]}$ Besides the critical question of the validity of the theoretical model for the description of spin relaxation, ${ }^{[14]}$ a satisfactory analysis of the experimental data is hampered by the large number of parameters entering the models. A more reliable data analysis is obtained by performing a simultaneous fit of all experimental data together ${ }^{[15]}$ but nevertheless, the correlation between certain parameters remains high in general.

If we consider only gadolinium-based compounds, which make up the majority of $T_{1}$ relaxivity contrast agents, the following classes of parameters can be distinguished: ${ }^{[7]}$

- parameters describing structural properties of the complexes, such as the number of first-sphere water molecules $(q)$, the distance between the gadolinium ion and water protons $\left(r_{\mathrm{GdH}}\right)$, the closest distance of approach for outersphere water molecules $(d)$;

- parameters describing the dynamics of Gd complexes in aqueous solution such as, for example, the rotational correlation time $\left(\tau_{\mathrm{R}}\right)$, correlation times for internal motion within the complex, relative translational diffusion between bulk water molecules and the complex $(D)$, exchange rate constants (or mean residence times $\tau_{\mathrm{M}}$ ) for water exchange from the first coordination sphere $\left(k_{\mathrm{ex}}=1 / \tau_{\mathrm{M}}\right)$;

- parameters describing the electron spin relaxation such as the strength of static $\left(\Delta_{\mathrm{s}}\right.$ or $D$ and $E$ ) and transient $\left(\Delta_{\mathrm{f}}\right)$ zero-field splitting and the correlation times for its fluctuations $\left(\tau_{\mathrm{R}}, \tau_{\mathrm{v}}\right)$.

To these parameters, one has to add various activation parameters like activation energies, enthalpies and entropies to describe the variation of the observed quantities with temperature. All together, the total number of parameters can exceed 25, depending on the model.

One possibility for decreasing the number of fitted parameters is the determination of some of them by computational means. Fossheim et al. studied, already in 1991, the structure-stability relationships of gadolinium(III) ion complexes by molecular mechanics (MM) calculations and molecular dynamics (MD) simulations. ${ }^{[16]}$ Determination of water coordination numbers $(q)$ by MM investigations have allowed to predict relaxivities on the basis of correlations to literature values. ${ }^{[17]}$ Studies of conformational equilibria of gadolinium polyaminocarboxylate complexes have been appearing since the end of the nineties. ${ }^{[18-21]}$ The dynamics of Gd complexes, including that of the first- and secondcoordination-sphere water molecules, has been investigated by classical MD simulations. ${ }^{[21,22]}$ Quantum mechanical calculations for $\mathrm{Gd}^{3+}$ have been performed in most cases by using pseudopotentials including the unpaired $4 f$ electrons within a frozen core. ${ }^{[23,24]}$ Calculations including the seven $f$ electrons of $\mathrm{Gd}^{3+}$ explicitly are more demanding and have only been carried out more recently. ${ }^{[25-31]}$ The use of smallcore pseudopotentials or even all-electron relativistic basis sets is essential if hyperfine interactions between the electron spin and the nuclear spin of surrounding atoms is to be calculated. ${ }^{[32]}$

In this microreview, we will mainly concentrate on our own work and consider computational studies of aqueous solutions of the gadolinium ion and its complexes that have a relation to MRI contrast agents.

\section{Classical Molecular Dynamics Simulations}

Simulations using classical, empirical force fields to describe intra- and intermolecular interactions have the advantage of being "cheap" in computer time needed. Therefore, long simulations of metal complexes in periodic boxes containing several hundreds of water molecules can be performed over several nanoseconds of simulation time. These long simulations allow studies of dynamics of the complex as well as the surrounding water molecules. However, the results depend strongly on the quality of the force field used. In simulations containing highly charged ions like lan-

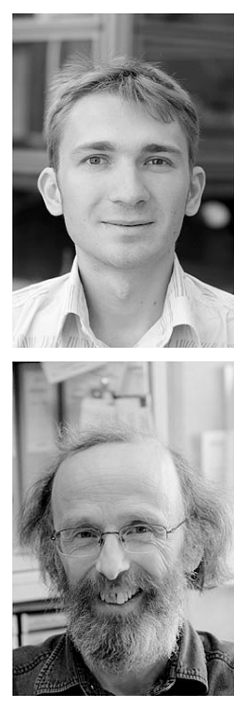

Oleg V. Yazyev was born in Simferopol, Ukraine, in 1981. He obtained his MSc degree in chemistry from the M. V. Lomonosov Moscow State University, Russian Federation, in 2003. In 2003 he started his PhD Thesis work under the supervision of Prof. Lothar Helm at the Ecole Polytechnique Fédérale de Lausanne (EPFL) and obtained his PhD degree in chemistry and chemical engineering in 2007. He is now a postdoctoral fellow at the Institute of Theoretical Physics (ITP) and the Institute for Numerical Research in the Physics of Materials (IRRMA) of the same institution. The scope of his theoretical research includes magnetic resonance imaging contrast agents, carbon nanostructures, and semiconductor surfaces and interfaces. 
thanide $3+$ cations, simple force fields including only van der Waals and Coulomb intermolecular interactions can lead to incorrect results. ${ }^{[27,28,33]}$ Including the polarization of molecules can partially correct these errors; however, this leads to a substantial increase in CPU time.

Another way to avoid errors due to the strong polarization is to consider the ion together with its first coordination sphere and to study the interaction of this entity with the surrounding water molecules. ${ }^{[34,35]}$ This "hydrated ion" approach $^{[36]}$ can be used if the first coordination sphere is inert in the sense that any exchange process is slow compared to the simulation time. Examples for hydrated ions studied by using this model are $\left[\mathrm{Cr}\left(\mathrm{H}_{2} \mathrm{O}\right)_{6}\right]^{3+},[34,36]$ $\left[\mathrm{Rh}\left(\mathrm{H}_{2} \mathrm{O}\right)_{6}\right]^{3+[37]}$ and $\left[\mathrm{Pt}\left(\mathrm{H}_{2} \mathrm{O}\right)_{4}\right]^{2+} \cdot{ }^{[38]}$

The water exchange rates from many polyaminocarboxylate complexes are in the order of $10^{6} \mathrm{~s}^{-1}$ and therefore slow enough for these complexes to be considered as essentially rigid units. ${ }^{[39]} \mathrm{MD}$ simulations using several Gd complexes with point charges optimized to reproduce the electrostatic potential on the surface and simple rigid TIP3P water ${ }^{[40]}$ molecules enabled the reproduction of experimental NMRD profiles. ${ }^{[22]}$ The simulations revealed that two to seven second-sphere water molecules are hydrogen-bound to the hydrophilic groups on the chelating ligands like carboxylates and phosphonates. Estimated residence times are of the order of 20-25 ps for polyaminocarboxylate and 56 ps for polyaminophosphonate ligands.

The coordination geometries of two macrocyclic and two acyclic $\mathrm{Gd}^{3+}$ complexes (Scheme 1) have been explored by classical MD with the use of atomic point charges on the ligand atoms obtained from ab initio calculations. ${ }^{[20,21]}$ Fast conformational changes of the acyclic complexes with DTPA $^{5-}$ and EGTA ${ }^{4-}$ have been found, whereas the macrocyclic complexes of DOTA ${ }^{4}$ and $\mathrm{DO}^{-} \mathrm{A}^{3-}$ were highly rigid: during simulations of $1 \mathrm{~ns}$, only small oscillations around the initial solid-state structures have been observed.
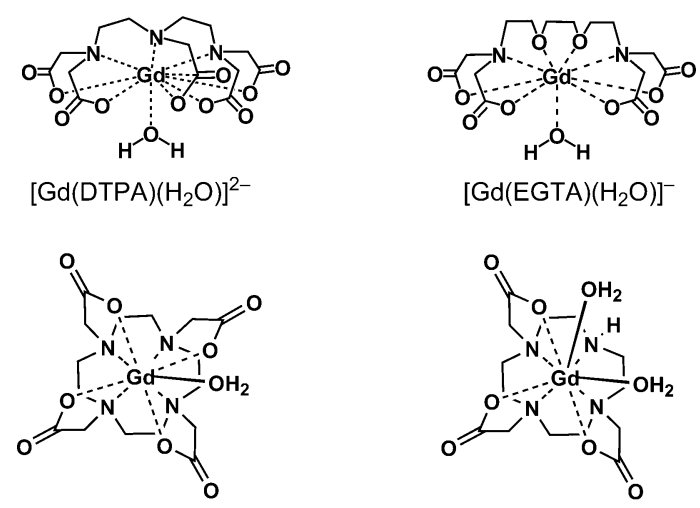

$\left[\mathrm{Gd}(\mathrm{DOTA})\left(\mathrm{H}_{2} \mathrm{O}\right)\right]^{-}$

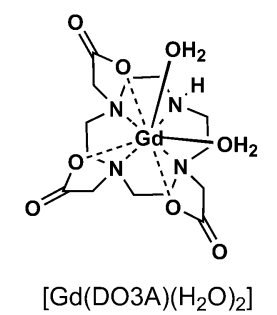

Scheme 1. Chemical structures of $\mathrm{Gd}^{3+}$ complexes with acyclic (top) and macrocyclic (bottom) chelating ligands.

An important parameter for the efficiency of MRI contrast agents is the rotational correlation time in solution. ${ }^{[41]}$ The rotational movement of Gd-based contrast agents can be investigated experimentally either by measuring the magnetic field dependence of the relaxation enhancement of water protons (NMRD profiles) or by measuring the longitudinal relaxation of the water ${ }^{17} \mathrm{O}$ nuclei as a function of temperature. ${ }^{[7]}$ The diffusive rotational motion of molecules in solution can be studied at a molecular level by MD simulations. The tumbling motion of the vectors either linking the paramagnetic centre to the bound water protons $\left(\mathbf{r}_{\mathrm{GdH}}\right)$ or pointing to the water oxygen $\left(\mathbf{r}_{\mathrm{GdO}}\right)$ (Figure 1$)$ can be established individually from the simulated trajectories. $\mathrm{Nu}$ clear spin relaxation rates are associated to the second-order correlation times, $\tau_{\mathrm{R}}=1 /\left(6 D_{\mathrm{R}}\right)$, where $D_{\mathrm{R}}$ is the rotational diffusion constant. ${ }^{[2]}$

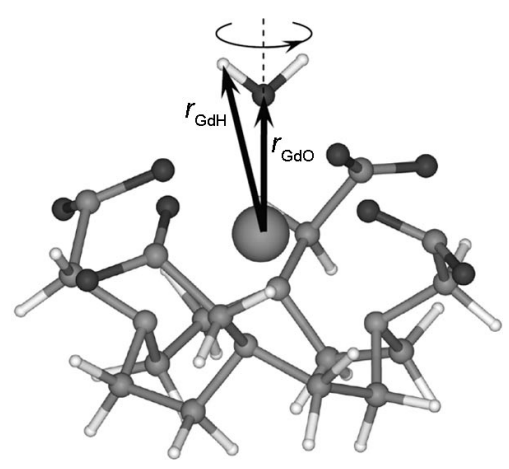

Figure 1. Definition of gadolinium-water vectors and the model for internal motion of a first-sphere water molecule.

The calculated correlation times for ${ }^{1} \mathrm{H}$ relaxation, $\tau_{\mathrm{R}}\left(\mathbf{r}_{\mathrm{GdH}}\right)$, are always shorter than those for ${ }^{17} \mathrm{O}$ relaxation, $\tau_{\mathrm{R}}\left(\mathbf{r}_{\mathrm{GdO}}\right)$ (Table 1). This can be explained for example by an "internal rotation" of the bound water molecule around the Gd-O axis (Figure 1). From the ratios $\tau_{\mathrm{R}}\left(\mathbf{r}_{\mathrm{GdH}}\right) / \tau_{\mathrm{R}}\left(\mathbf{r}_{\mathrm{GdO}}\right)$, it follows that this internal motion is faster for the complexes with acyclic ligands DTPA and EGTA than for the complexes with macrocyclic DOTA and DO3A. From simple geometrical models it can be shown that, even for the case of an extremely fast hypothetical internal rotation of the inner-sphere water molecule, the effective rotational correlation time for $\mathbf{r}_{\mathrm{GdH}}$ is only about $35 \%$ shorter than that for $\mathbf{r}_{\mathrm{GdO}}{ }^{[44]}$ The correlation time for the rotational motion of the whole complex, $\tau_{\mathrm{R}}$ (complex), can be obtained from the sum of all Gd-A vectors, where A are all binding atoms (N and $\mathrm{O}$ ) of the macrocyclic or acyclic ligands. ${ }^{[20]}$ The values of $\tau_{\mathrm{R}}$ (complex) for all complexes studied are close to those

Table 1. Calculated second-order rotational correlation times of simulated $\mathrm{Gd}^{3+}$ complexes.

\begin{tabular}{lcccc}
\hline & DOTA $^{4-}$ & DO3A $^{3-}$ & DTPA $^{5-}$ & EGTA $^{4-}$ \\
\hline$\tau_{\mathrm{R}}\left(\mathbf{r}_{\mathrm{GdO}}\right)[\mathrm{ps}]$ & 51 & 36,33 & 50 & 41 \\
$\tau_{\mathrm{R}}\left(\mathbf{r}_{\mathrm{GdH}}\right)^{\mathrm{aa}]}[\mathrm{ps}]$ & 41 & 27 & 32 & 31 \\
$\tau_{\mathrm{R}}($ complex $)[\mathrm{ps}]$ & 52 & 37 & 50 & 43 \\
$\tau_{\mathrm{R}}\left(\mathbf{r}_{\mathrm{GdH}}\right) / \tau_{\mathrm{R}}\left(\mathbf{r}_{\mathrm{GdO}}\right)$ & 0.82 & 0.82 & 0.64 & 0.72 \\
$\tau_{\mathrm{R}}(\exp ).[\mathrm{ps}]$ & $77^{[\mathrm{b}]}$ & $66^{[\mathrm{c}]}$ & $58^{[\mathrm{b}]}$ & $58^{[\mathrm{d}]}$ \\
\hline
\end{tabular}

[a] Mean value for both protons of bound water molecules. [b] Ref. $^{[15]}[\mathrm{c}]$ Ref. $^{[42]}$ [d] Ref. ${ }^{[43]}$ 
for the $\mathrm{Gd}-\mathrm{O}$ vector linking the central ion to the water oxygen. Experimental correlation times, $\tau_{\mathrm{R}}(\exp$.), are always longer than the calculated ones. In the simulation, an infinite dilution of the complex is assumed, and the ionic strength is zero, which is clearly not the case in experiments.

\section{Quadrupolar Relaxation - Electric Field Gradients}

The rotational correlation time of the above-defined vector, $\mathbf{r}_{\mathrm{GdO}}$, can experimentally be obtained from the longitudinal relaxation rates, $1 / T_{1}$, of oxygen-17 NMR spectroscopy. ${ }^{[7]}$ For water molecules in the first coordination sphere of $\mathrm{Gd}^{3+}$, this relaxation rate is the sum of a dipolar contribution, due to interaction with the electron spin of the paramagnetic centre, and of a quadrupolar contribution, because ${ }^{17} \mathrm{O}$ has a nuclear spin $I=5 / 2$ and therefore an electric quadrupole moment, $Q$. The quadrupole relaxation term, $1 / T_{1 Q}$, is in the case of relatively fast rotation (extreme narrowing condition) directly linked to the rotational correlation time $\tau_{\mathrm{R}}$ [Equation (1)]. ${ }^{[2,45,46]}$

$\frac{1}{T_{1 Q}}=\frac{4 \pi^{2}}{10} \frac{2 I+3}{I^{2}(2 I-1)} \chi^{2}\left(1+\frac{\eta^{2}}{3}\right) \tau_{\mathrm{R}}$

The quadrupole coupling constant $\chi$ and the asymmetry parameter $\eta$ are given by the electric field gradient at the oxygen nucleus and the electric quadrupole moment. ${ }^{[46]}$ Knowledge of $\chi$ and $\eta$ therefore allows a direct determination of $\tau_{\mathrm{R}}$, provided that the dipolar contribution to $1 / T_{1}$ is known or small. The electric field gradient tensor, $\mathbf{V}$, is linked to the local environment of the oxygen atom and can be calculated by using quantum chemical methods. ${ }^{[47-49]}$ Quadrupole coupling constants of oxygen-17 have been determined for neat water, but only few, sometimes contradictory estimations are available for $\mathrm{H}_{2} \mathrm{O}$ molecules in the first coordination sphere of the cation. ${ }^{[44,50-53]}$

Model calculations on $\mathrm{Gd}-\mathrm{H}_{2} \mathrm{O}$ clusters have been used to assess the dependence of ${ }^{17} \mathrm{O}$ quadrupole coupling parameters on the $\mathrm{Gd}-\mathrm{O}$ distance and on the orientation of the water dipole vector of the first-sphere water molecules (tilt angle). ${ }^{[54]}$ Only slight changes of $\chi$ and $\eta$ with the $\mathrm{Gd}-$ $\mathrm{O}$ distance have been found. The dependence on the tilt angle $(\theta)$ is, however, more pronounced: $\chi$ decreases by more than $20 \%$ if $\theta$ is increased from $0^{\circ}$ to $90^{\circ}$. At the same time, the asymmetry parameter, $\eta$, increases from 0.6 to almost 1 . The orientation of the principal vectors of the electric field gradient tensor, $\mathbf{V}$, do not change significantly: the largest component $\left(V_{\mathrm{zz}}\right)$ which is linked to $\chi$ is always perpendicular to the plane of the water molecule (Figure 2).

To obtain realistic values of quadrupole coupling parameters in liquids, statistical averages have to be calculated. This can be achieved by a conjunction of molecular dynamics simulations with quantum chemical calculations. ${ }^{[49,55-57]}$ MD simulations (Figure 3) can be performed by using one of the empirical force fields available or with a first-prin-

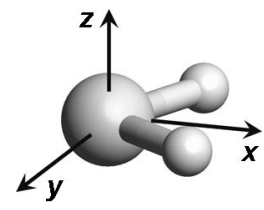

Figure 2. Convention on the orientation of principal axes of the ${ }^{17} \mathrm{O}$ quadrupole coupling tensor $\mathbf{V}$ in a water molecule.

ciples MD method such as the density-functional-theorybased Car-Parrinello molecular dynamics (CPMD) method. ${ }^{[58]}$ On one hand, density functional theory tends to overstructure liquid water, ${ }^{[59,60]}$ and on the other hand, CPMD is more suitable for the description of coordination compounds than classical MD based on empirical force fields.

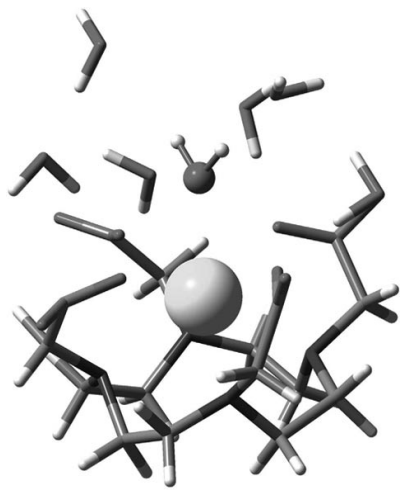

Figure 3. A typical snapshot extracted from a classical MD simulation of $\left[\mathrm{Gd}(\mathrm{DOTA})\left(\mathrm{H}_{2} \mathrm{O}\right)\right]^{-}$. The $\mathrm{Gd}^{3+}$ ion and the first-sphere water molecule are shown in balls and sticks; the DOTA ligand and six second-coordination-sphere water molecules are presented as tubes.

We performed classical MD simulations (TIP3P) ${ }^{[40]}$ of neat water, $\mathrm{Gd}^{3+}$ dissolved in water and $\left[\mathrm{Gd}(\mathrm{DOTA})\left(\mathrm{H}_{2} \mathrm{O}\right)\right]^{-}$ in aqueous solution as well as CPMD calculations for the first two. ${ }^{[54]}$ From the trajectories of the simulations, we extracted clusters in a way that the water molecule for which electric field gradient tensors are calculated is surrounded by at least one full coordination sphere. For the $\mathrm{Gd}^{3+}$ aqua ion, for example, clusters contained one ion surrounded with the 24 closest water molecules. The time interval between extracted snapshots in classical MD simulation is in the order of several picoseconds. ${ }^{[54]}$ Because of the limited duration of the CPMD simulations, the time interval is equal to $0.25 \mathrm{ps}$ in these cases. Such a short time interval is nevertheless larger than the characteristic times of both vibrations of inner-sphere water molecules and their "wagging" motions, which also exhibit a high degree of broadening of the corresponding power spectrum band. ${ }^{[61]}$ Statistical analysis of the calculated properties showed no sizeable time correlation. We thus conclude that the sampling of quadrupole and hyperfine coupling parameters over the short trajectories used here is able to provide accurate ensemble averages (Table 2). The statistical errors given in this work are standard deviations of the mean. 
Table 2. Calculated ${ }^{17} \mathrm{O}$ quadrupole coupling parameters.

\begin{tabular}{lcccc}
\hline & $\begin{array}{c}\chi \\
{[\mathrm{MHz}]}\end{array}$ & $\eta$ & $\begin{array}{c}\chi\left(1+\eta^{2} / 3\right)^{1 / 2} \\
{[\mathrm{MHz}]}\end{array}$ \\
\hline Neat liquid $\mathrm{H}_{2} \mathrm{O}$ & TIP3P $^{[a]}$ & $8.38 \pm 0.07$ & $0.66 \pm 0.01$ & $8.98 \pm 0.08$ \\
& CPMD $^{[b]}$ & $8.11 \pm 0.08$ & $0.82 \pm 0.01$ & $8.98 \pm 0.08$ \\
{$\left[\mathrm{Gd}\left(\mathrm{H}_{2} \mathrm{O}\right)_{8}\right]^{3+}$} & TIP3P $^{[a]}$ & $9.06 \pm 0.06$ & $0.60 \pm 0.01$ & $9.59 \pm 0.07$ \\
& CPMD $^{[b]}$ & $8.23 \pm 0.06$ & $0.72 \pm 0.01$ & $8.91 \pm 0.06$ \\
{$\left[\mathrm{Ca}\left(\mathrm{H}_{2} \mathrm{O}\right)_{6}\right]^{2+}$} & CPMD $^{[b]}$ & $8.1 \pm 0.2$ & $0.78 \pm 0.03$ & $8.9 \pm 0.2$ \\
{$\left[\mathrm{Gd}(\mathrm{DOTA})\left(\mathrm{H}_{2} \mathrm{O}\right)\right]^{-}$} & TIP3P $^{[a]}$ & $8.42 \pm 0.06$ & $0.46 \pm 0.01$ & $8.72 \pm 0.05$ \\
\hline
\end{tabular}

[a] From classical MD simulations by using TIP3P water. [b] From first-principles MD by using CPMD.

Figure 4 shows the distributions of $\chi, \eta$ and $\chi(1+$ $\left.\eta^{2} / 3\right)^{1 / 2}$ together with the mean values calculated. A significant difference between the results based on classical MD and CPMD simulations is only observed for the asymmetry parameter $\eta=\left(V_{y y}-V_{x x}\right) / V_{z z}$. The shift to higher values is consistent with the overall overstructuring of the DFT water. The results from classical simulations of [Gd$\left.\left(\mathrm{H}_{2} \mathrm{O}\right)_{8}\right]^{3+}$ are biased because of the rather low quality of the force field used and the lack of polarization. ${ }^{[54]}$ All other quadrupole coupling parameters are very close, and it has been concluded that ${ }^{17} \mathrm{O}$ quadrupole coupling parameters water molecules in the neat liquid state.
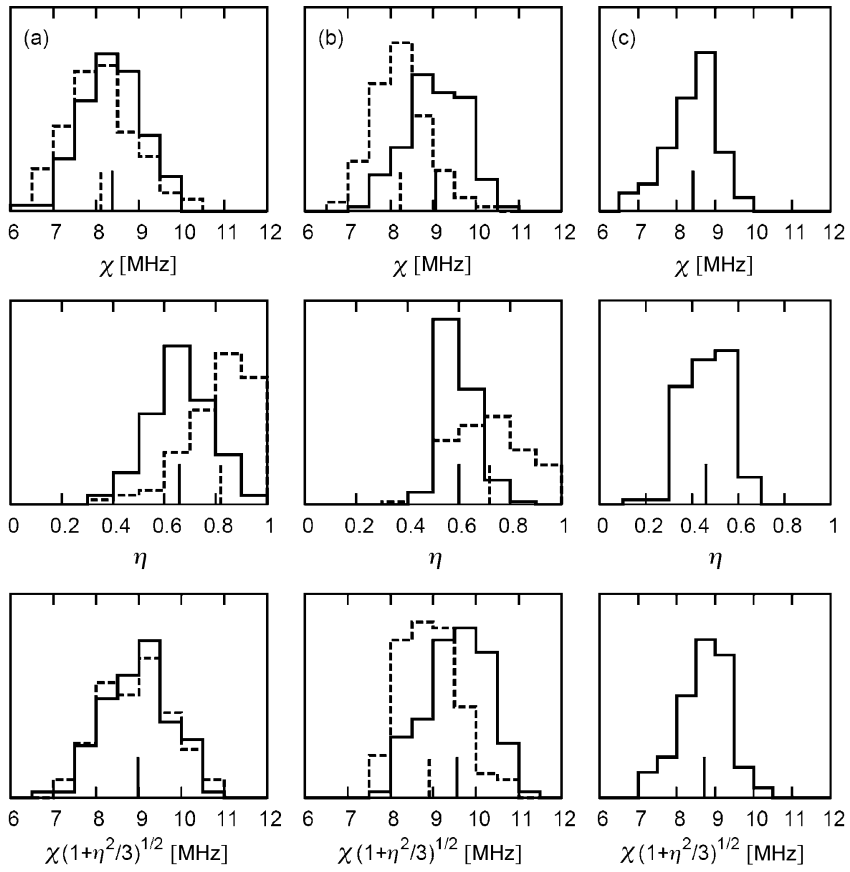

Figure 4. Histogram plots of the distribution of the ${ }^{17} \mathrm{O}$ quadrupole coupling parameters calculated for $\left(\mathrm{H}_{2} \mathrm{O}\right)_{18}$ clusters (a), first-coordination-sphere water molecules of $\left[\mathrm{Gd}\left(\mathrm{H}_{2} \mathrm{O}\right)_{8}\right]^{3+}$ (b) and [Gd(DOTA) $\left.\left(\mathrm{H}_{2} \mathrm{O}\right)\right]^{-}$(c) sampled from classical (solid line) and CPMD simulations (dashed line). Vertical lines indicate corresponding mean values.

\section{Hyperfine Interactions}

Hyperfine interactions (HFI) between the electron spin of paramagnetic ions and the nuclear spin of ligand atoms are the driving force for NMR relaxation enhancement of solvent nuclei in solutions containing paramagnetic species $^{[62,63]}$ and therefore also for relaxivity of MRI contrast agents. ${ }^{[64]}$ These interactions are directly linked to the spin distribution $\rho^{\alpha-\beta}(\mathbf{r})=\rho^{\alpha}(\mathbf{r})-\rho^{\beta}(\mathbf{r})$ at the location of the nucleus considered. In many cases, the spin density distribution is determined by the shapes of singly occupied molecular orbitals (SOMOs). This contribution is usually called "spin delocalization" and it is always positive if $\rho^{\alpha}(\mathbf{r})$ is the density of the majority spin $\alpha$ and $\rho^{\beta}(\mathbf{r})$ is the density of the minority spin $\beta$. A second part of the spin density comes from so called spin-polarization effects, which originate from unequal potentials experienced by $a$ and $\beta$ electrons and orthogonality constraints imposed on molecular orbitals (MOs): this leads to different shapes of $a$ and $\beta$ MOs (which would otherwise be doubly occupied). The spin-polarization contribution can be positive as well as negative at the location of a nucleus $\mathrm{N}$, but it always integrates to zero over space. Spin-polarization effects can be described in short as an "effective attraction": the localized unpaired electrons "attract" the nearby ones of the same spin. This can result in a negative spin density in the vicinity of SOMO nodes and where SOMO density is vanishing and in a slight increase in positive spin density, produced by the spin-delocalization effect. Spin polarization is often referred to as a second-order effect. ${ }^{[65]}$ The superposition of spin delocalization and spin polarization will be referred to as spin distribution from now on.

Quantum chemical calculations can provide some a priori knowledge on HFI between paramagnetic ions and ligand nuclear spins as has been shown by Kowalewski for $d$ transition-metal ions more than twenty years ago. ${ }^{[66,67]}$ The ligand HFI in complexes containing an $f$-transition-metal ion like a lanthanide is different from that in complexes of $d$-transition-metal ions. Due to the core character of the $f$ shell, any significant contribution of ligand atomic orbitals to singly occupied molecular orbitals (SOMOs) is improbable. ${ }^{[25]}$ However, a negative spin-polarization effect has been calculated for the $f^{7}$ ion $\mathrm{Gd}^{3+}$ in complexes with one $\mathrm{H}_{2} \mathrm{O}$ or $\mathrm{NH}_{3}$ molecule. [68]

Hyperfine interactions are magnetic interactions, and the corresponding spin Hamiltonian can be written as shown in Equation (2): ${ }^{[69]}$

$\mathbf{H}=\mathbf{S} \cdot \mathbf{A} \cdot \mathbf{I}$

where $\mathbf{A}$ is the $3 \times 3$ HFI tensor, and $\mathbf{S}$ and $\mathbf{I}$ are the vectors of electron and nuclear spin, respectively. The HFI tensor is commonly split into an isotropic part and an anisotropic part, as given by Equation (3):

$\mathbf{A}=A_{\text {iso }} \mathbf{1}+\mathbf{T}$

where $A_{\text {iso }}$ is the isotropic hyperfine coupling constant (scalar), $\mathbf{1}$ is the $3 \times 3$ unit matrix and $\mathbf{T}$ is the traceless matrix of the anisotropic contribution. With the commonly used Breit-Pauli approximation, the scalar isotropic (Fermi contact) HFI constant at nucleus $\mathrm{N}$ is expressed by Equation (4): ${ }^{[70]}$ 
$A_{\text {iso }}(\mathrm{N})=\frac{4 \pi}{3 S} \beta_{\mathrm{e}} \beta_{\mathrm{N}} g_{\mathrm{e}} g_{\mathrm{N}} \rho^{\alpha-\beta}\left(\mathbf{R}_{\mathrm{N}}\right)$

where $\beta_{\mathrm{e}}\left(9.2740 \times 10^{-24} \mathrm{JT}^{-1}\right)$ and $\beta_{\mathrm{N}}\left(5.0507 \times 10^{-27} \mathrm{JT}^{-1}\right)$ are the Bohr and the nuclear magnetons, respectively, $g_{\mathrm{e}}$ and $g_{\mathrm{N}}$ are the free-electron and nuclear $g$ values and $\mathbf{S}$ is the maximum value of the electron spin projection. ${ }^{[65]}$ The matrix elements of the anisotropic (dipolar) contribution can be calculated from Equation (5):

$$
\begin{aligned}
& T_{i j}(\mathrm{~N})=\frac{1}{2 S} \beta_{\mathrm{e}} \beta_{\mathrm{N}} g_{\mathrm{e}} g_{\mathrm{N}} \int \rho^{\alpha-\beta}(\mathbf{r}) \times \\
& \frac{\left(\mathbf{r}-\mathbf{R}_{\mathrm{N}}\right)^{2} \delta_{i j}-3\left(r_{i}-R_{\mathrm{N}}\right)\left(r_{j}-R_{\mathrm{N}_{j}}\right)}{\left(\mathbf{r}-\mathbf{R}_{\mathrm{N}}\right)^{5}} \mathrm{~d} \mathbf{r}
\end{aligned}
$$

where $\mathbf{R}_{\mathrm{N}}$ is the position of the nucleus $\mathrm{N}$ in space relative to the paramagnetic centre. The isotropic contribution, also called Fermi contact or scalar contribution, $A_{\text {iso }}$, is proportional to the value of the spin density at the position of nucleus $\mathrm{N}$, and therefore it possesses a local character. Physically this contribution represents a magnetic field generated at the point of the nucleus by the presence of the electron magnetic moment itself. On the contrary, the anisotropic contribution, $\mathbf{T}$, is the dipolar integral over the whole space and has therefore a nonlocal character. It represents the dipole-dipole type of magnetic interaction between the magnetic moments of nuclear and electron spins. The dipolar contribution vanishes if the spin density is highly symmetric when observed from the point of nucleus $\mathrm{N}^{\left[{ }^{[32]}\right.}$

The key step in the quantum chemical calculation of hyperfine interactions is the calculation of the spin distribution $\rho^{\alpha-\beta}(\mathbf{r})$. Calculations for compounds containing lanthanides or other heavy elements require an adequate treatment of relativistic effects. ${ }^{[2,71]}$ Usually one has to choose between an all-electron treatment and relativistic effective core potentials.

The reliability of density functional calculations of HFI for $\mathrm{Gd}^{3+}$ complexes has been assessed by calculations on the small model complex $\left[\mathrm{Gd}\left(\mathrm{H}_{2} \mathrm{O}\right)_{8}\right]^{3+}$ (Figure 5). ${ }^{[32]}$ The calculations showed that both pure (BPW91) and hybrid (B3PW91) density functionals look reliable enough to describe HFI of ligand nuclei in the model compound. From the spin density map of $\left[\mathrm{Gd}\left(\mathrm{H}_{2} \mathrm{O}\right)_{8}\right]^{3+}$ (Figure 6) it can be seen that most of the positive electron spin density resides on the $\mathrm{Gd}^{3+}$ ion itself, but a significant spin density due to spin-polarization exists along the $\mathrm{Gd}-\mathrm{O}$ axis.

To calculate ligand hyperfine interactions of hydrated ions and ion complexes in aqueous solution, a similar approach has been applied as for electric field gradient calculations. Clusters of molecules were extracted from trajectories generated by either classical or first-principles molecular dynamics simulations. In the following we will first discuss the results of scalar hyperfine interaction calculations and then the results of the calculations of dipolar (anisotropic) HFI. Nuclear spins of ligand atoms directly bound to a paramagnetic centre are in general mostly affected by scalar hyperfine interaction. These ligand atoms are mainly oxy-

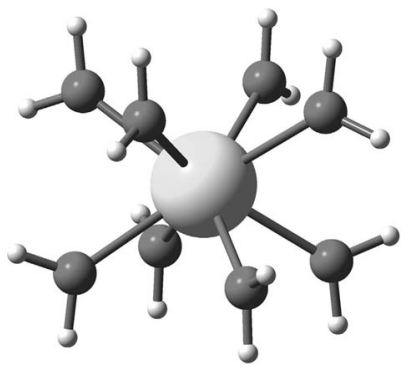

Figure 5. Structure of the model $\mathrm{Gd}^{3+}$ octaaqua complex of $D_{4 d}$ symmetry.

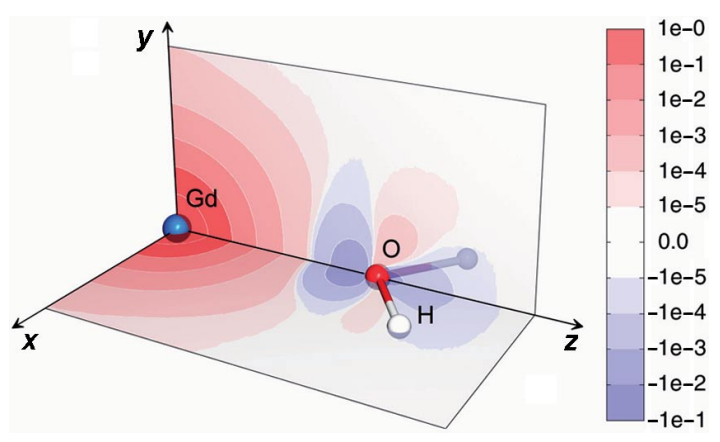

Figure 6. Spin density map of the $\left[\mathrm{Gd}\left(\mathrm{H}_{2} \mathrm{O}\right)_{8}\right]^{3+}$ model system (calculated at the BPW91/DKH2/Hirao level of theory, in a.u. ${ }^{-3}$ ) showing the $\mathrm{Gd}^{3+}$ ion and one of the eight water molecules. Cross sections of $2 \times 4 \AA$ in size in the $x \mathrm{O} z$ and $y \mathrm{O} z$ planes are shown.

gen, nitrogen and sulfur, all having NMR-active isotopes with a relatively low gyromagnetic ratio $\gamma$. Protons with a larger $\gamma$ are in general further away from the paramagnetic ion and their spin relaxation is often dominated by the dipolar interaction (see below).

\section{Scalar Hyperfine Interaction}

The scalar hyperfine interaction results in a strong shift of the NMR resonance frequency, $\delta^{\mathrm{SC}}$, of directly bound atoms and in an enhancement of the longitudinal and especially the transverse relaxation rates $1 / T_{1}{ }^{\mathrm{SC}}$ and $1 / T_{2} \mathrm{SC}$, respectively. In particular, the enhancement of $1 / T_{2}$ can be used to measure the rate constant, $k_{\mathrm{ex}}$, of very fast solvent exchange reactions via the scalar relaxation mechanism [Equation (6)]. ${ }^{[72,73]}$

$\frac{1}{T_{2}^{\mathrm{sC}}}=\frac{S(S+1)}{3}\left(\frac{A}{\hbar}\right)^{2}\left[\tau_{\mathrm{sl}}+\frac{\tau_{\mathrm{s} 2}}{1+\omega_{\mathrm{S}}^{2} \tau_{\mathrm{s} 2}^{2}}\right] ; \tau_{\mathrm{si}}^{-1}=T_{i \mathrm{e}}^{-1}+k_{\mathrm{ex}}$

The scalar coupling constant $A / \mathrm{h}$ as defined in Equation (6) is equal to $2 \pi A_{\text {iso }}$ as defined in Equation (4), $T_{\text {ie }}(i=1$, 2) are the electron spin relaxation times, $k_{\mathrm{ex}}$ is the exchange rate constant for the exchange of a specific water molecule $^{[74]}$ and $\omega_{\mathrm{S}}=\left(g_{\mathrm{e}} \beta_{\mathrm{e}} / \hbar\right) B_{0}$ is the electron spin resonance frequency corresponding to the magnetic field $B_{0}$ applied 
for the NMR experiment. The scalar coupling constant $A / \hbar$ can be obtained from chemical shift measurements [Equation (7)], but these experiments are hampered by susceptibility effects and give only average values for molecules in the first and second coordination sphere. ${ }^{[7]}$

$\delta^{\mathrm{SC}}=\frac{g_{\mathrm{e}} \beta_{\mathrm{e}} S(S+1)}{3 k_{B} T \gamma_{I}}\left(\frac{A}{\hbar}\right)$

To start, quantum chemical calculations of the scalar coupling constant have been performed on the hydrated $\mathrm{Gd}^{3+}$ ion in aqueous solution. Figure 7 shows a snapshot from the Car-Parrinello MD simulation performed in a periodic box of volume $V=(11.648 \AA)^{3}$ containing one $\mathrm{Gd}^{3+}$ ion and 56 water molecules. ${ }^{[61]}$ Spatial regions with positive (red) and negative (blue) spin density can be distinguished. The spin-polarization pattern of the eight innersphere water molecules is similar to the one calculated on the model complex (Figure 5). Calculated scalar HFI coupling constants of first- and second-sphere water molecules are given in Table 3. The negative spin polarization at the location of the first-sphere water oxygen corresponds to a positive $A_{\text {iso }}\left({ }^{17} \mathrm{O}\right)$ due to the negative nuclear gyromagnetic ratio of ${ }^{17} \mathrm{O}, \gamma_{17 \mathrm{O}}=g_{17 \mathrm{O}} \beta_{\mathrm{N}} / \hbar<0$. First-sphere $A_{\text {iso }}$ values for ${ }^{17} \mathrm{O}$ obtained from CPMD snapshots show a very strong distance dependence (Figure 8, left) but the average value is very similar to that obtained with the model cluster $\left[\mathrm{Gd}\left(\mathrm{H}_{2} \mathrm{O}\right)_{8}\right]^{3+} \cdot{ }^{[32]}$ This is probably a consequence of the very similar $\mathrm{Gd}-\mathrm{O}$ distance in both systems $(2.4 \AA$ for the model cluster and an average distance of $2.37 \AA$ for the CPMD simulation). Most recent experimental values of $A_{\text {iso }}=A / h$ are $0.83 \mathrm{MHz}^{[75]}$ and $0.84 \mathrm{MHz}^{[15]}$ from NMR shift measurements and $0.75 \mathrm{MHz}^{[53]}$ from ENDOR experiments.

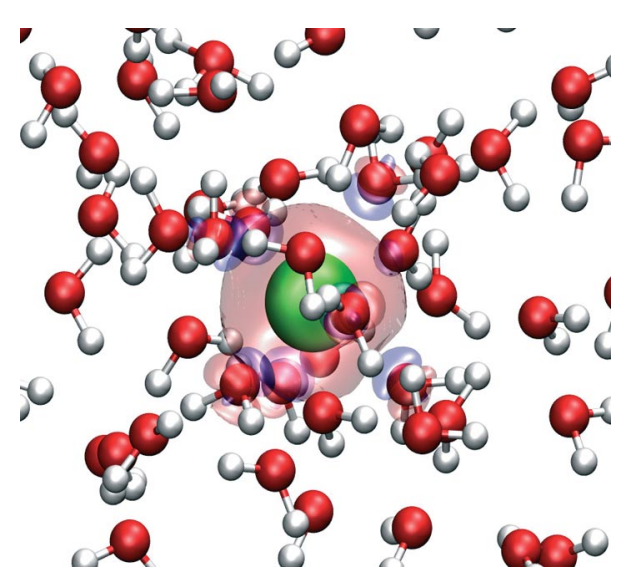

Figure 7. Atomic structure and isosurface plot of the spin density around $\mathrm{Gd}^{3+}$ ion in solution. Red surfaces correspond to 0.0002 a.u. $^{-3}$, blue surfaces correspond to -0.0002 a.u. ${ }^{-3}$ values.

The inner-sphere ${ }^{1} \mathrm{H}$ hyperfine coupling constant shows a similar negative correlation with distance (Figure 8, right). The mean value is larger than experimental values available $\left(0.03 \pm 0.02 \mathrm{MHz},{ }^{[76]} 0.04 \mathrm{MHz}^{[77]}\right)$ as well as the
Table 3. Comparison of calculated isotropic hyperfine coupling constants for ${ }^{17} \mathrm{O}$ and ${ }^{1} \mathrm{H} \cdot{ }^{[32,61,78]}$.

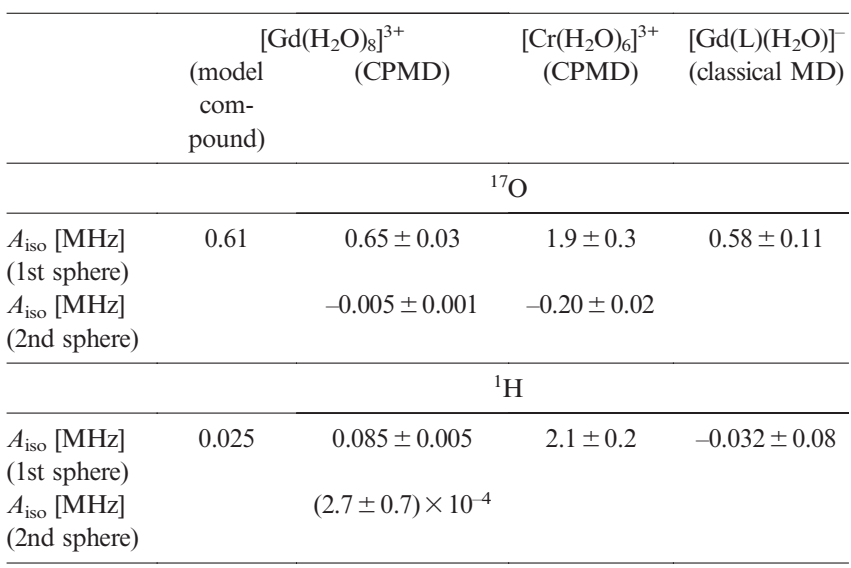
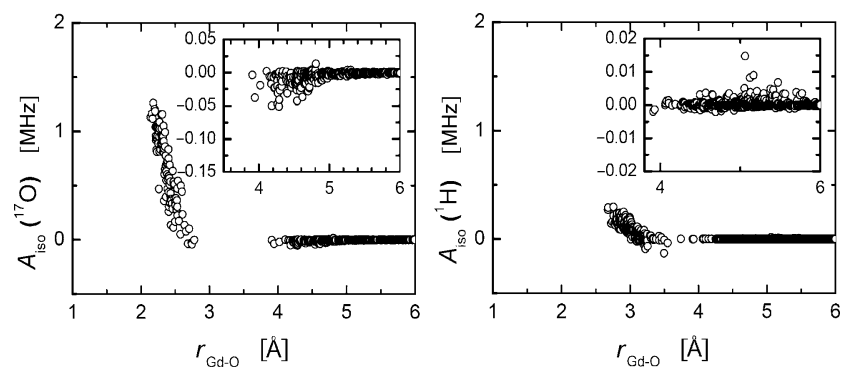

Figure 8. $\mathrm{Gd}^{3+}$ in aqueous solution: isotropic scalar coupling constant, $A_{\text {iso }}$, for ${ }^{17} \mathrm{O}$ (left) and for ${ }^{1} \mathrm{H}$ (right) both as a function of $\mathrm{Gd}-\mathrm{O}$ distance.

value obtained from the model cluster. However, experimental and calculated $A_{\text {iso }}\left({ }^{1} \mathrm{H}\right)$ values as well as their differences are small.

Spin densities (Figure 9) calculated from the CPMD snapshots of $\left[\mathrm{Gd}\left(\mathrm{H}_{2} \mathrm{O}\right)_{8}\right]^{3+}$ in solution allowed scalar hyperfine coupling constants for second-sphere water molecules to be obtained. The absolute average values for secondsphere $A_{\text {iso }}\left({ }^{17} \mathrm{O}\right)$ and for $A_{\text {iso }}\left({ }^{1} \mathrm{H}\right)$ are more than two orders of magnitude smaller than corresponding first-sphere values (Table 3). Interestingly, there is a change in sign for $A_{\text {iso }}\left({ }^{17} \mathrm{O}\right)$ : mean spin polarization is positive at secondsphere water oxygen positions and negative at first-sphere water oxygen positions. Second-sphere scalar coupling for ${ }^{1} \mathrm{H}$ spins as calculated from the CPMD trajectory is negligible.

A big difference in absolute values of first and secondsphere $A_{\text {iso }}$ has also been found for the hydrated $\mathrm{Cr}^{3+}$ ion, studied by using the same first-principles MD approach. ${ }^{[78]}$ For this $d$ transition metal ion, absolute magnitudes and inner-sphere/outer-sphere ratios of the scalar hyperfine coupling constants are larger (Table 3 ). This certainly reflects the strong interaction between the $d$ electrons of $\mathrm{Cr}^{3+}$ and water oxygen, leading also to a much more organized second coordination with very slow water exchange $\left(2.4 \times 10^{-6} \mathrm{~s}^{-1}\right) \cdot{ }^{[79]}$ The calculations confirmed the considerable spin delocalization on the second-coordination-sphere water oxygen atoms (Figure 10) for $\mathrm{Cr}^{3+}$. The average, 


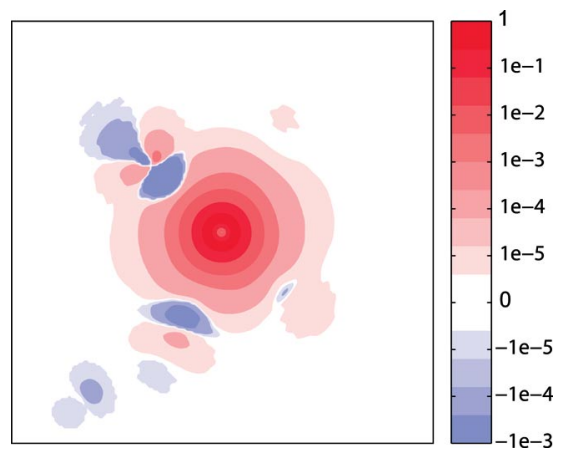

Figure 9. Contour plot of the spin-density distribution in a plane passing through the $\mathrm{Gd}^{3+}$ ion. An area of $10 \AA \times 10 \AA$ is shown. The $\mathrm{Gd}^{3+}$ ion located in the middle gives rise to a large positive magnetization density. The anisotropic spin polarization of four inner-sphere and one outer-sphere water molecules can also be recognized on the plot.

$\left\langle A_{\text {iso }}\left({ }^{17} \mathrm{O}_{\text {II }}\right)\right\rangle=-0.20 \mathrm{MHz}$, is in good agreement with the experimental value of $-0.215 \mathrm{MHz}^{[35]}$ Similarly to the octahydrated $\mathrm{Gd}^{3+}$, a change in sign from first to second-coordination sphere has also been found for the hexahydrated $\mathrm{Cr}^{3+}$. This change was observed experimentally for $\left[\mathrm{Ti}\left(\mathrm{H}_{2} \mathrm{O}\right)_{6}\right]^{3+}{ }^{[80]}$
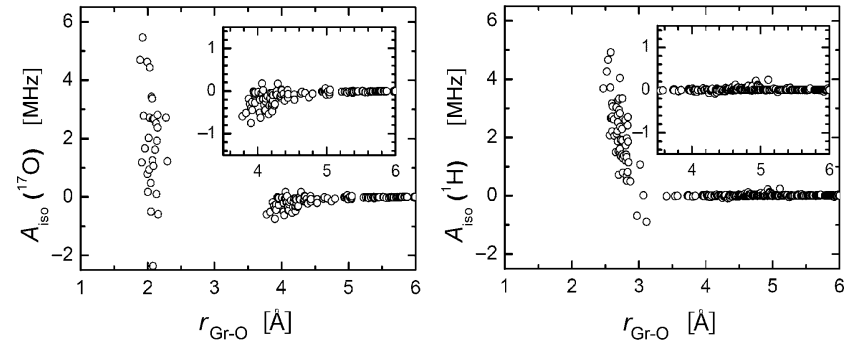

Figure $10 . \mathrm{Cr}^{3+}$ in aqueous solution: isotropic scalar coupling constant, $A_{\text {iso, }}$, for ${ }^{17} \mathrm{O}$ (left) and for ${ }^{1} \mathrm{H}$ (right) both as a function of $\mathrm{Cr}-\mathrm{O}$ distance.

The only polyaminocarboxylate complex of $\mathrm{Gd}^{3+}$ studied so far for hyperfine interaction by quantum chemical meth-

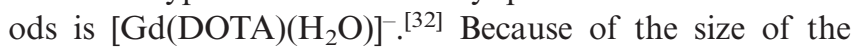
complex, only trajectories from a classical MD simulation are available for extraction of clusters. In all snapshots the DOTA ligand is in its major (M) conformation with approximate square antiprismatic geometry. ${ }^{[21,81]}$

The major parameter influencing the ${ }^{17} \mathrm{O}$ hyperfine coupling constant, $A_{\text {iso, }}$ is the gadolinium-oxygen distance, $r_{\mathrm{Gd}-\mathrm{O}}$, which varies during the 1-ns simulation from 2.44 to $2.7 \AA$ (Figure 11, left). ${ }^{[21]}$ The averaged $A_{\text {iso }}\left({ }^{17} \mathrm{O}\right)$ is $0.58 \pm 0.11 \mathrm{MHz}$ for an average distance of $2.56 \pm 0.06 \AA$, which is in excellent agreement with the value determined from chemical shift measurements $\left(0.59 \mathrm{MHz}^{[15]}\right)$. All 100 individual $A_{\text {iso }}\left({ }^{17} \mathrm{O}\right)$ values calculated form the snapshots are positive, which means that the spin density at the position of the oxygen is negative in accord with the spin-polarization mechanism. The $A_{\text {iso }}$ values for the water protons are distributed from $+0.16 \mathrm{MHz}$ to $-0.21 \mathrm{MHz}$ with a small negative average value of $-0.032 \pm 0.08 \mathrm{MHz}$ (Figure 11, right). As for ${ }^{17} \mathrm{O}$ a correlation with the distance to the $\mathrm{Gd}^{3+}$ ion is observed. Experimental values for $A_{\text {iso }}\left({ }^{1} \mathrm{H}\right)$ are
$+0.075 \mathrm{MHz}$ (from the NMR chemical shift) ${ }^{[82]}$ and $-0.04 \mathrm{MHz}$ measured by 2-dimensional Mims ENDOR on $\left[\mathrm{Gd}(\mathrm{HP}-\mathrm{DO} 3 \mathrm{~A})\left(\mathrm{H}_{2} \mathrm{O}\right)\right],{ }^{[77]}$ with an agreement which is satisfactory if the small absolute magnitude is considered.
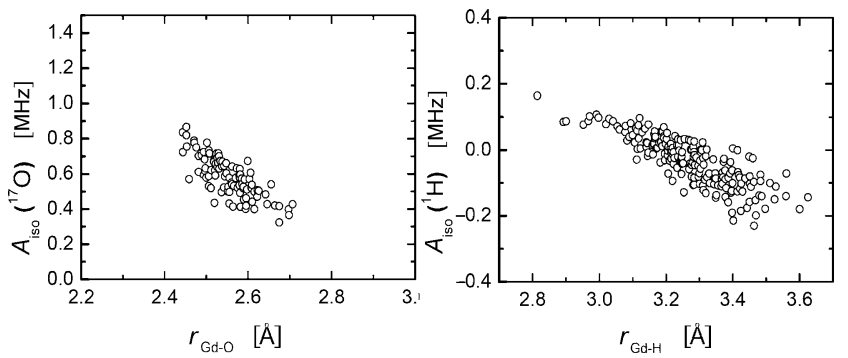

Figure 11. Hyperfine coupling constants $A_{\text {iso }}$ for inner-sphere water ${ }^{17} \mathrm{O}$ (left) and ${ }^{1} \mathrm{H}$ (right) of $\left[\mathrm{Gd}(\text { DOTA })\left(\mathrm{H}_{2} \mathrm{O}\right)\right]^{-}$calculated from classical MD snapshots and plotted as a function of $\mathrm{Gd}-\mathrm{O}$ and $\mathrm{Gd}-\mathrm{H}$ distances, respectively.

\section{Dipolar Hyperfine Interaction}

The anisotropic part of the hyperfine interaction between the paramagnetic centre and ${ }^{1} \mathrm{H}$ nuclear spins is the main source for $T_{1}$ relaxation enhancement induced by gadolinium-based MRI contrast agents. The resulting dipolar relaxation for bound water protons is commonly calculated by using the modified Solomon-Bloembergen-Morgan equations. ${ }^{[14,63,83]}$ These equations are based, among other approximations, on the point-dipole approximation. ${ }^{[69]}$ Other equations are available for slowly rotating complexes, taking into account a more adequate description of the electron spin relaxation due to zero-field splitting. ${ }^{[14,84,85]}$ Nevertheless, all of them are still based on the point-dipole approximation for dipolar interaction, and they neglect spin-distribution effects. The dipolar ligand HFI tensor, $\mathbf{T}^{\mathrm{PD}}$, corresponding to this approximation depends only on the distance between the metal and the ligand nuclei, $r_{\mathrm{MX}}$. Assuming that the metal-ligand nucleus vector, $\mathbf{r}_{\mathrm{MX}}$, is $\left(r_{\mathrm{MX}}, 0,0\right)$, it follows that [Equation (8)]:

$\mathbf{T}^{\mathrm{PD}}=\beta_{\mathrm{e}} \beta_{\mathrm{N}} g_{\mathrm{e}} g_{\mathrm{N}} \frac{1}{r_{\mathrm{MX}}^{3}}\left(\begin{array}{ccc}2 & 0 & 0 \\ 0 & -1 & 0 \\ 0 & 0 & -1\end{array}\right)$

From the anisotropic part $\mathbf{T}$ of the HFI tensor [Equation (3)], we can calculate an effective distance $r_{\text {eff }}$ for the dipole-dipole interaction [Equation (9)]:[67,69]

$r_{\mathrm{eff}}=\left(\frac{S}{\beta_{\mathrm{e}} \beta_{\mathrm{N}} g_{\mathrm{e}} g_{\mathrm{N}}}\right)^{2}\left[T_{z z}^{2}+\frac{1}{3}\left(T_{x x}-T_{y y}\right)^{2}\right]^{-1 / 6}$

where $T_{z z}$ is the maximal absolute value of the principal components of $\mathbf{T}$, and $T_{x x}$ and $T_{y y}$ are its other two eigenvalues. If the electron spin density is strictly localized on the paramagnetic ion itself, which is the case in the pointdipole approximation, $r_{\text {eff }}$ is equal to the internuclear dis- 
tance $r_{\mathrm{MX}}$. As a result of the spin polarization of water molecules in direct contact with the ion, either an enhancement or a screening of the dipolar interaction between electron and nuclear spin can be observed. To visualize eventual deviations from the point-dipole approximation, we calculated the enhancement factors, $\left(r_{\mathrm{MX}} / r_{\mathrm{eff}}\right)^{3}$, for dipolar interaction involving ${ }^{17} \mathrm{O}$ and ${ }^{1} \mathrm{H}$ nuclear spins and $\mathrm{Gd}^{3+}$ from snapshots of a CPMD simulation of $\left[\mathrm{Gd}\left(\mathrm{H}_{2} \mathrm{O}\right)_{8}\right]^{3+}$ in aqueous solution (Figure 12). ${ }^{[61]}$
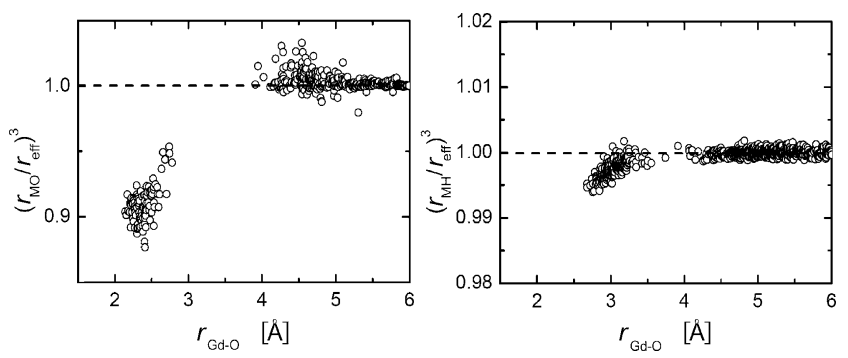

Figure 12. Dipolar hyperfine interaction enhancement factors $\left(r_{\mathrm{MX}} / r_{\mathrm{eff}}\right)^{3}$ for ${ }^{17} \mathrm{O}$ nuclei (left) and for ${ }^{1} \mathrm{H}$ nuclei (right) as a function of the $\mathrm{Gd}-\mathrm{O}$ distance calculated for hydrated $\mathrm{Gd}^{3+}$ ions in aqueous solution.

The ${ }^{17} \mathrm{O}$ dipolar hyperfine interactions of first-sphere water molecules $\left(r_{\mathrm{Gd}-\mathrm{O}}<3 \AA\right)$ are partially screened as a result of spin polarization, leading to an average value of $\left\langle\left(r_{\mathrm{MO}} / r_{\mathrm{eff}}\right)^{3}\right\rangle=0.91 \pm 0.1$. A clear increase in the screening is observed if the water oxygen approaches the $\mathrm{Gd}^{3+}$ ion (Figure 12, left). The dipolar interactions of ${ }^{17} \mathrm{O}$ nuclei of second-coordination-sphere water molecules (from the radial distribution function: $\left.3.8 \AA<r_{\mathrm{Gd}-\mathrm{O}}<5.2 \AA\right)^{[61]}$ are slightly enhanced with respect to what is expected form the point-dipole approximation. Screening of the ${ }^{1} \mathrm{H}$ dipolar hyperfine interaction of inner-sphere water is small, $\left\langle\left(r_{\mathrm{MH}}\right\rangle\right.$ $\left.\left.r_{\text {eff }}\right)^{3}\right\rangle=0.995 \pm 0.001$, and the effects of spin polarization on the dipolar interaction on outer-sphere water molecules are negligible (Figure 12, right).

Anisotropic hyperfine coupling constants of $\mathrm{H}_{2} \mathrm{O}$ nuclear spins in $\left[\mathrm{Gd}(\mathrm{DOTA})\left(\mathrm{H}_{2} \mathrm{O}\right)\right]^{-}$have been calculated from snapshots of a classical MD trajectory as described above. The dependence on the $\mathrm{Gd}-\mathrm{O}$ distance of the enhancement factors $\left(r_{\mathrm{MO}} / r_{\mathrm{eff}}\right)^{3}$ for ${ }^{17} \mathrm{O}$ is much less pronounced in the case of the DOTA complex as compared to $\left[\mathrm{Gd}\left(\mathrm{H}_{2} \mathrm{O}\right)_{8}\right]^{3+}$ (Figure 12 and Figure 13). The average calculated effective $\mathrm{Gd}-\mathrm{O}$ distance for dipolar interaction, $r_{\text {eff }}$, is $2.72 \pm 0.05 \AA$, which is about $6 \%$ longer than the average internuclear distance. Because dipolar spin relaxation depends on distance by $1 / r^{6}$, deviation from the point-dipole approximation is considerably higher: the averages calculated on the 6th power of $1 / r$ lead for ${ }^{17} \mathrm{O}$ to a $31 \%$ lower relaxation relative to the point-dipole approximation relaxation. It has to be kept in mind that the total ${ }^{17} \mathrm{O}$ longitudinal relaxation of water bound to $\mathrm{Gd}^{3+}$ is the sum of contributions due to dipolar and quadrupolar relaxation (see above).

The effective distance of the dipole-dipole interaction of ${ }^{1} \mathrm{H}$ spins deviates only slightly from the internuclear $\mathrm{Gd}-\mathrm{H}$ distance, $r_{\mathrm{Gd}-\mathrm{H}}$ (Figure 13, right). The influence on ${ }^{1} \mathrm{H}$ spin relaxation is only about $2 \%$ for the closest protons, which
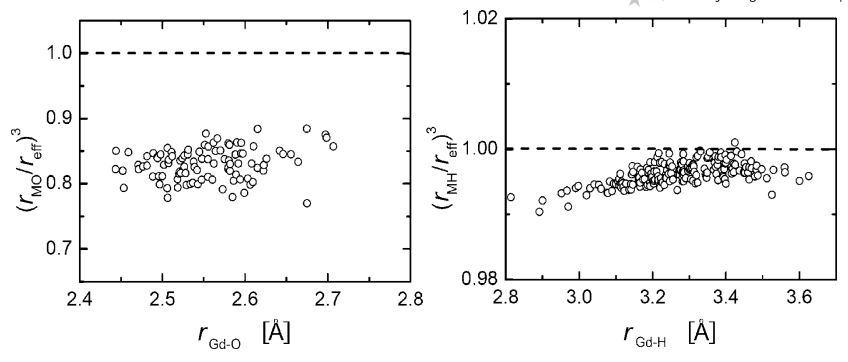

Figure 13. Dipolar hyperfine interaction enhancement factors $\left(r_{\mathrm{MX}} / r_{\text {eff }}\right)^{3}$ for ${ }^{17} \mathrm{O}$ nuclei (left) and ${ }^{1} \mathrm{H}$ nuclei (right) as a function of $\mathrm{Gd}-\mathrm{O}$ and $\mathrm{Gd}-\mathrm{H}$ distances for inner-sphere water in $[\mathrm{Gd}-$ (DOTA) $\left.\left(\mathrm{H}_{2} \mathrm{O}\right)\right]^{-}$.

implies that the point-dipole approximation is valid for describing proton relaxation in gadolinium-based contrast agents.

\section{Conclusions}

In recent years it could be shown that quantum chemical calculations, together either with classical or with first-principles molecular dynamics simulations, can provide a link between measured nuclear spin relaxation rates and the structure and dynamics of compounds at a molecular level. Simulations of the dynamics is still restricted to either results from simulations based on empirical force fields or on relatively small systems on short time scales in the case of quantum-chemistry-based simulations.

Structure-based calculations either for electric field gradients for the quadrupolar relaxation mechanism or for hyperfine interactions for the scalar and dipolar relaxation mechanisms can provide quantitative information on parameters entering equations for calculation of relaxation rates. Values for solutions can be obtained by statistical averaging in combining quantum chemical calculations with liquids simulations. These calculations reduce the number of freely adjustable parameters for the fitting of experimental data or, in a less favourable case, allow the restriction of these parameters to a small range.

Another valuable outcome of the calculations is testing of approximations in the evaluation of nuclear spin relaxation. An important approximation, the point-dipole approximation, is for example a very good one for ${ }^{1} \mathrm{H}$ relaxation, but is relatively limited for spin of directly bound atoms like ${ }^{17} \mathrm{O}$.

Further developments for quantum mechanical calculations in relation to relaxation phenomena in paramagnetic systems in solution would be for electron spin relaxation. Calculation of parameters for zero-field splitting for lanthanide ions would be important for the synthesis of new MRI contrast agents with slow electron spin relaxation and thus with high relaxivities.

\section{Acknowledgments}

This work was supported by the Swiss National Science Foundation and the Swiss State Secretariat for Education and Research - 
SER. The research was carried out in the frame of the EU COST Action D38 and European founded EMIL program LSHC-2004503569 .

[1] N. Bloembergen, E. M. Purcell, R. V. Pound, Phys. Rev. 1948, $73,679-712$

[2] A. Abragam, The Principles of Nuclear Magnetism, Clarendon Press, Oxford, 1961.

[3] R. M. Weisskoff, R. R. Edelman in Basic Principles of MRI, Vol. 1 (Eds.: R. R. Edelman, J. R. Hesselink, M. B. Zlatkin, V. Crues III), Saunders, Philadelphia, 1996, pp. 3-51.

[4] N. E. Brasch, West. J. Med. 1985, 142, 847-853.

[5] H. J. Weinmann, R. C. Brasch, W. R. Press, G. E. Wesbey, Am. J. Roentgenol. 1984, 142, 619-624.

[6] A. E. Merbach, É. Tóth, The Chemistry of Contrast Agents in Medical Magnetic Resonance Imaging, John Wiley \& Sons, Chichester, 2001.

[7] É. Tóth, L. Helm, A. E. Merbach in Relaxivity of Gadolinium(III) Complexes: Theory and Mechanism (Eds.: A. E. Merbach, E. Tóth), John Wiley \& Sons, Chichester, 2001, pp. 45119.

[8] R. B. Lauffer, Chem. Rev. 1987, 87, 901-927.

[9] S. H. Koenig, R. D. Brown III, Prog. NMR Spectrosc. 1990, 22, 487-567.

[10] R. Van Eldik, I. Bertini, Relaxometry of Water-Metal Ion Interactions, Elsevier, San Diego, 2005.

[11] P. Caravan, J. J. Ellison, T. J. McMurry, R. B. Lauffer, Chem. Rev. 1999, 99, 2293-2352.

[12] L. Vander Elst, F. Maton, S. Laurent, F. Seghi, F. Chapelle, R. N. Muller, Magn. Res. Medicine 1997, 38, 604-614.

[13] A. Borel, L. Helm in Very High Frequency (VHF) ESR/EPR (Eds.: O. Grinberg, L. J. Berliner), Springer, New York, 2004, vol. 22 , pp. $207-247$

[14] J. Kowalewski, D. Kruk, G. Parigi in NMR Relaxation in Solution of Paramagnetic Complexes: Recent Theoretical Progress for $S \geq 1$, Adv. Inorg. Chem., vol. 57 (Eds.: R. Van Eldik, I. Bertini), Elsevier, San Diego, 2005, pp. 42-104.

[15] D. H. Powell, O. M. Ni Dhubhghaill, D. Pubanz, L. Helm, Y. S. Lebedev, W. Schlaepfer, A. E. Merbach, J. Am. Chem. Soc. 1996, 118, 9333-9346.

[16] R. Fossheim, H. Dugstad, S. G. Dahl, J. Med. Chem. 1991, 34, 819-826.

[17] D. E. Reichert, R. D. Hancock, M. J. Welch, Inorg. Chem 1996, 35, 7013-7020.

[18] U. Cosentino, G. Moro, D. Pitea, A. Villa, P. C. Fantucci, A. Maiocchi, F. Uggeri, J. Phys. Chem. A 1998, 102, 4606-4614.

[19] U. Cosentino, D. Pitea, G. Moro, V. Barone, A. Villa, R. N. Muller, F. Botteman, Theor. Chem. Acc. 2003, 111, 204-209.

[20] F. Yerly, K. I. Hardcastle, L. Helm, S. Aime, M. Botta, A. E. Merbach, Chem. Eur. J. 2002, 8, 1031-1039.

[21] F. Yerly, A. Borel, L. Helm, A. E. Merbach, Chem. Eur. J. 2003, 9, 5468-5480.

[22] A. Borel, L. Helm, A. E. Merbach, Chem. Eur. J. 2001, 7, 600610

[23] M. Dolg, H. Stoll, H. Preuss, Theor. Chem. Acc. 1993, 85, 441450.

[24] M. Dolg in Electronic Structure Calculations for Molecules Containing Lanthanide Ions, Handbook on the Physics and Chemistry of Rare Earths, vol. 22 (Eds.: K. A. Gscheidner, L. Eyring), Elsevier, Amsterdam, 1996, pp. 3-116.

[25] L. Maron, O. Eisenstein, J. Phys. Chem. A 2000, 104, 71407143.

[26] X. Cao, M. Dolg, J. Chem. Phys. 2001, 115, 7348-7355.

[27] C. Clavaguera, E. Sansot, F. Calvo, J.-P. Dognon, J. Phys. Chem. B 2006, 110, 12848-12851.

[28] C. Clavaguera, F. Calvo, J. P. Dognon, J. Chem. Phys. 2006, 124, 074505 .

[29] C. Clavaguera, J. P. Dognon, P. Pyykkö, Chem. Phys. Lett. 2006, 429, $8-12$
[30] R. Pollet, C. Clavaguera, J. P. Dognon, J. Chem. Phys. 2006, 124,164103

[31] R. Pollet, D. Marx, J. Chem. Phys. 2007, 126, 181102.

[32] O. V. Yazyev, L. Helm, V. G. Malkin, O. L. Malkina, J. Phys. Chem. A 2005, 109, 10997-11005.

[33] T. Kowall, F. Foglia, L. Helm, A. E. Merbach, J. Am. Chem. Soc. 1995, 117, 3790-3799.

[34] R. R. Pappalardo, J. M. Martinez, E. S. Marcos, J. Phys. Chem. 1996, 100, 11748-11754

[35] A. Bleuzen, F. Foglia, E. Furet, L. Helm, A. E. Merbach, J. Weber, J. Am. Chem. Soc. 1996, 118, 12777-12787.

[36] J. M. Martinez, R. R. Pappalardo, E. S. Marcos, J. Chem. Phys. 1998, 109, 1445-1455.

[37] J. M. Martinez, P. Merkling, R. R. Pappalardo, K. Refson, E. S. Marcos, Theor. Chem. Acc. 2004, 111, 101-109.

[38] F. Torrico, R. R. Pappalardo, E. Sanchez Marcos, J. M. Martinez, Theor. Chem. Acc. 2006, 115, 196-203.

[39] L. Helm, A. E. Merbach, Chem. Rev. 2005, 105, 1923-1960.

[40] W. L. Jorgensen, J. Chandrasekhar, J. D. Madura, J. Chem. Phys. 1983, 79, 926-935.

[41] S. Aime, M. Botta, E. Terreno in $G d^{I I I}$-Based Contrast Agents for MRI, Adv. Inorg. Chem., vol. 57 (Eds.: R. Van Eldik, I. Bertini), Elsevier, San Diego, 2005, pp. 173-237.

[42] S. Aime, M. Botta, S. G. Crich, G. B. Giovenzana, R. Pagliarin, M. Sisti, E. Terreno, Magn. Reson. Chem. 1998, 38, S200-S208.

[43] S. Aime, A. Barge, A. Borel, M. Botta, S. Chemerisov, A. E. Merbach, U. Müller, D. Pubanz, Inorg. Chem. 1997, 36, 5104 5112.

[44] F. A. Dunand, A. Borel, A. E. Merbach, J. Am. Chem. Soc. 2002, 124, 710-716.

[45] T. E. Bull, S. Forsén, D. L. Turner, J. Chem. Phys. 1979, 70, 3106-3111.

[46] B. Halle, H. Wennerström, J. Chem. Phys. 1981, 75, 1928-1943.

[47] H. P. Huber, A. J. Dyson, B. Kirchner, Chem. Soc. Rev. 1999, $28,121-133$

[48] R. Ludwig, F. Weinhold, T. C. Farrar, J. Chem. Phys. 1996 , $105,8223-8230$.

[49] H. P. Huber, Z. Naturforsch., Teil A 1994, 49, 103-115.

[50] P. L. Cummins, G. B. Bacskay, N. S. Hush, B. Halle, S. Engström, J. Chem. Phys. 1985, 82, 2002-2013.

[51] R. P. W. J. Struis, J. de Bleijser, J. C. Leyte, J. Phys. Chem. 1987, 91, 6309-6315.

[52] X. Tan, M. Bernardo, H. Thomann, C. P. Scholes, J. Chem. Phys. 1995, 102, 2675-2690.

[53] A. M. Raitsimring, A. V. Astashkin, D. Baute, D. Goldfarb, P. Caravan, J. Phys. Chem. A 2004, 108, 7318-7323.

[54] O. V. Yazyev, L. Helm, J. Chem. Phys. 2006, 125, 054503.

[55] R. Eggenberger, S. Gerber, H. P. Huber, D. J. Searles, M. Welker, J. Comput. Chem. 1993, 14, 1553-1560.

[56] R. Eggenberger, S. Gerber, H. P. Huber, D. J. Searles, M. Welker, Mol. Phys. 1993, 80, 1177-1182.

[57] T. S. Pennanen, J. Vaara, P. Lantto, A. J. Sillanpaepae, K. Laasonen, J. Jokisaari, J. Am. Chem. Soc. 2004, 126, 11093-11102.

[58] R. Car, M. Parrinello, Phys. Rev. Lett. 1985, 55, 2471-2474.

[59] I.-F. W. Kuo, C. J. Mundy, M. J. McGrath, J. I. Siepmann, J. VandeVondele, M. Sprik, J. Hutter, B. Chen, M. L. Knlein, F. Mohammed, M. Krack, M. Parrinello, J. Phys. Chem. B 2004, 108, 12990-12998.

[60] Y. A. Mantz, B. Chen, G. J. Martyna, J. Phys. Chem. B 2005 , $110,3540-3554$

[61] O. V. Yazyev, L. Helm, J. Chem. Phys. 2007, 127, 084506.

[62] I. Bertini, C. Luchinat, Coord. Chem. Rev. 1996, 150, 1-296.

[63] I. Bertini, C. Luchinat, G. Parigi, Solution NMR of Paramagnetic Molecules, Elsevier, Amsterdam, 2001.

[64] L. Helm, É. Tóth, A. E. Merbach in Lanthanide Ions as Magnetic Resonance Imaging Agents. Nuclear and Electronic Relaxation Properties. Applications., Lanthanides and Their Interrelations with Biosystems, vol. 40 (Eds.: H. Sigel, A. Sigel), Marcel Dekker, Inc., New York, 2003, pp. 589-641. 
[65] M. Kaupp, M. Bühl, V. G. Malkin, Calculation of NMR and EPR Parameters Theory and Applications, Wiley-VCH, Weinheim, 2004.

[66] J. Kowalewski, A. Laaksonen, L. Nordenskiöld, M. Blomberg, J. Chem. Phys. 1981, 74, 2927-2930.

[67] L. Nordenskiöld, A. Laaksonen, J. Kowalewski, J. Am. Chem. Soc. 1982, 104, 379-382.

[68] E. D. Glendening, P. A. Petillo, J. Phys. Chem. B 2001, 105, 1489-1493.

[69] J. Kowalewski, L. Nordenskiöld, N. Benetis, P. Westlund, Prog. in NMR Spectroscopy 1985, 17, 141-185.

[70] To convert from atomic units used in quantum chemical calculations to SI units, values have to be multiplied by $\mu_{0}(4 \pi h)^{-1}$.

[71] P. Pyykkö, Chem. Rev. 1988, 88, 563-594.

[72] K. Micskei, D. H. Powell, L. Helm, E. Brücher, A. E. Merbach, Magn. Reson. Chem. 1993, 31, 1011-1020.

[73] P. Caravan, A. E. Merbach, Chem. Commun. 1997, 2147-2148.

[74] S. F. Lincoln, A. E. Merbach, Adv. Inorg. Chem. 1995, 42, 188.

[75] A. Borel, F. Yerly, L. Helm, A. E. Merbach, J. Am. Chem. Soc. 2002, 124, 2042-2048.
[76] J. Reuben, D. Fiat, J. Chem. Phys. 1969, 51, 4909-4917.

[77] A. V. Astashkin, A. M. Raitsimring, P. Caravan, J. Phys. Chem. A 2004, 108, 1990-2001.

[78] O. V. Yazyev, L. Helm, Theor. Chem. Acc. 2006, 115, 190-195.

[79] F. C. Xu, H. R. Krouse, T. W. Swaddle, Inorg. Chem. 1985, 24, 267-270.

[80] A. D. Hugi, L. Helm, A. E. Merbach, Inorg. Chem. 1987, 26, $1763-1768$.

[81] S. Aime, M. Botta, M. Fasano, M. P. M. Marques, C. Geraldes, D. Pubanz, A. E. Merbach, Inorg. Chem. 1997, 36, 2059-2068.

[82] C. Bryden, C. N. Reilley, J. F. Desreux, Anal. Chem. 1981, 53, $1418-1425$.

[83] J. Kowalewski, L. Mäler, Nuclear Spin Relaxation in Liquids: Theory, Experiments, and Applications, Taylor \& Francis, New York, 2006.

[84] S. Rast, P. H. Fries, E. Belorizky, J. Chem. Phys. 2000, 113, 8724-8735.

[85] D. Kruk, Theory of Evolution and Relaxation in Multi-Spin Systems, Abramis, Suffolk, 2007.

Received: September 19, 2007

Published Online: December 7, 2007 